\title{
Effects of expressing lamin A mutant protein causing Emery-Dreifuss muscular dystrophy and familial partial lipodystrophy in HeLa cells
}

\author{
Kim Bechert, Mariana Lagos-Quintana, Jens Harborth, Klaus Weber, and Mary Osborn* \\ Max Planck Institute for Biophysical Chemistry, Department of Biochemistry, Am Fassberg 11, 37077 Göttingen, Germany
}

Received 30 September 2002, revised version received 28 November 2002

\begin{abstract}
Patients with the autosomal dominant form of Emery-Dreifuss muscular dystrophy (EDMD) or familial partial lipodystrophy (FPLD) have specific mutations in the lamin A gene. Three such point mutations, G465D (FPLD), R482L, (FPLD), or R527P (EDMD), were introduced by site-specific mutagenesis in the C-terminal tail domain of a FLAG-tagged full-length lamin A construct. HeLa cells were transfected with mutant and wild-type constructs. Lamin A accumulated in nuclear aggregates and the number of cells with aggregates increased with time after transfection. At $72 \mathrm{~h}$ post transfection $60-80 \%$ of cells transfected with the mutant lamin A constructs had aggregates, while only $35 \%$ of the cells transfected with wild-type lamin A revealed aggregates. Mutant transfected cells expressed $10-24 \times$, and wild-type transfected cells $20 \times$, the normal levels of lamin A. Lamins C, B1 and B2, Nup153, LAP2, and emerin were recruited into aggregates, resulting in a decrease of these proteins at the nuclear rim. Aggregates were also characterized by electron microscopy and found to be preferentially associated with the inner nuclear membrane. Aggregates from mutant constructs were larger than those formed by the wild-type constructs, both in immunofluorescence and electron microscopy. The combined results suggest that aggregate formation is in part due to overexpression, but that there are also mutant-specific effects.
\end{abstract}

(C) 2003 Elsevier Science (USA). All rights reserved.

Keywords: Lamin A; Lamins; Emery-Dreifuss muscular dystrophy; Familial partial lipodystrophy; Nuclear aggregates; Emerin

\section{Introduction}

Mutations in the lamin A gene have been shown to be responsible for six human diseases. The myopathies include dilated cardiomyopathy (DCM) with conduction system defects [1], limb girdle muscular dystrophy 1B with atrioventricular conduction disturbances (LGMD1B) [2], and the autosomal dominant form of Emery-Dreifuss muscular dystrophy (EDMD) $[3,4]$. This latter disease is characterized by early contractures of the ankles, elbows, and Achilles' tendons, slowly progressive muscle wasting, and weakness and in some cases cardiomyopathy with conduction block [5]. The fourth disease is Dunnigan-type familial partial lipodystrophy (FPLD) [6-8]. Patients suffering from FPLD show complete or partial absence of adipose tissues from the extremeties and trunk after puberty [9]. Additional dis-

\footnotetext{
* Corresponding author. Fax: +49-551-201-1578.

E-mail address: mosborn@gwdg.de (M. Osborn).
}

eases associated with mutations in the lamin A gene are autosomal recessive axonal neuropathy (Charcot-MarieTooth disorder type 2) [10] characterized by muscle weakness and wasting, foot deformities, and electrophysiological as well as histological changes, and mandibuloacral dysplasia, a rare autosomal recessive disorder characterized by postnatal growth retardation, craniofacial anomalies, skeletal malformations, and mottled cutaneous pigmentation [11]. Mutations causing each of the above diseases have been mapped along the lamin A gene (reviewed by Genschel and Schmidt [12] and Hutchison et al. [13]).

Lamins $\mathrm{A}$ and $\mathrm{C}$ arise from alternatively spliced forms of the lamin A gene and diverge only at their C-termini [1416]. Expression of the lamin A/C gene is developmentally regulated. In mice lamin $\mathrm{A} / \mathrm{C}$ expression is not found in cells of the early embryo or in undifferentiated teratocarcinoma cell lines. It is detected in the embryo proper first at embryonic day 12 in certain muscle cells, but begins to be expressed only at 15 days after birth in brain $[17,18]$. Stem 
cells of the immune and hemopoietic systems lack lamin $\mathrm{A} / \mathrm{C}$ even in adult animals [19]. In contrast, lamins B1 and B2 are encoded by two distinct genes and are thought to be constituitively expressed irrespective of developmental stage [20-22] and, indeed, B-type lamins are found at all stages of mouse development [18]. A third B-type laminlamin B3-is a splice variant of lamin B2 and its expression is limited to male germ cells [23].

Knockout of the lamin A gene in mice results in mice that at birth cannot be distinguished from wild-type, except for some redistribution of emerin, a protein of the inner nuclear membrane. However, such mice show severely retarded postnatal growth, develop muscular dystrophy, and die at 6-8 weeks of age. Thus, the pathology of the lamin A knockout mice is strikingly similar to that seen with human EDMD [24]. RNA interference experiments on lamins in human HeLa cells have shown that silencing of the lamin A gene did not affect cell growth, but did lead to a redistribution of emerin. These results show that lamin A is not an essential protein in HeLa cells. In contrast, lamin B1 and lamin B2 are essential since silencing of these genes leads to apoptosis [25].

The nuclear lamina underlies the inner membrane of the nuclear envelope [26]. This 10-20-nm layer is built of polymers formed by the nuclear lamins, which are most probably arranged as a net, although this ultrastructural feature has so far only been demonstrated for the Xenopus oocyte [27]. It is thought to play a critical role in maintaining the structural integrity of the nuclear envelope, but may also have additional functions. The lamina is connected to the nuclear envelope by lamin-binding proteins that are integral membrane proteins of the inner nuclear membrane. This latter class includes three isoforms of lamina-associated protein 1 (LAPI A, B, and C), at least five isoforms of LAP2, emerin, and the lamin B receptor [28,29]. A sixth isoform of LAP2, LAP2 $\alpha$, lacks an inner nuclear membrane-binding domain [28]. Emerin binds specifically to A-type lamins both in vivo [30,24] and in vitro [31]. LAP1A and LAP1B specifically bind to lamin A, C, and B1 [32], while LAP2 $\alpha$ is associated exclusively with A-type lamins [33] and lamin B receptor interacts with B-type lamins [34]. The lamina is also associated with nuclear pore complexes perhaps through a tight association of nuclear lamins with Nup153, a peripheral nuclear pore component [35]. Interactions between the nuclear lamina and chromatin are thought to be mediated through the $\mathrm{C}$-terminal lamin sequences [36].

To understand how different mutations in the lamin A molecule can affect lamina assembly and nuclear organization, we and others have used additional approaches with cells in culture. In the first approach, primary cultures of fibroblasts from FPLD patients with heterozygous mutations in lamin A at position 482 were studied [37]. Dysmorphic nuclei were seen in fibroblasts from three patients. The frequency with which such abnormalities were observed varied between $5 \%$ and $22 \%$, depending on the patient and the passage number, while $2-4 \%$ of control fibroblasts had similar abnormalities. Equivalent studies have not yet been performed with cells from patients with the other three diseases. In a second approach, point mutations were inserted into the full-length lamin A cDNA and used to transfect cells in culture $[38,39]$. Changes were seen in the lamin A arrangement after transfecting HeLa cells with two point mutants that cause DCM and a single mutant that causes EDMD, but not with a point mutation causing FPLD [38]. In a second study, aggregate formation was seen in the lamin A arrangement after transfecting $\mathrm{C} 2 \mathrm{C} 12$ myoblasts with four mutants, one of which causes DCM and three of which cause EDMD [39]. No change was seen in the same study with 11 other mutant constructs, three of which cause DCM, five of which cause EDMD, and three cause FPLD. Our own interest in lamin A led us to make several mutant lamin A constructs that cause EDMD and FPLD in parallel to the studies reported by Östlund et al. [39] and Raharjo et al. [38]. Our results suggest that, as argued by others, there are mutant-specific effects. In addition, however, they give insight into lamin A aggregate formation. This phenomenon was reported by Östlund et al. [39] with mutant, but not with the wild-type, lamin A construct. Our data show that both the mutant and the wild-type lamin A constructs form aggregates. At any time point the mutant cultures have about twice as many transfected cells with aggregates as do the wild-type cultures. Other lamins, as well as several laminassociated proteins, are recruited into the aggregates. Aggregates have been characterized not only by immunofluorescence microscopy but also by electron microscopy, and the majority are shown to underlie the nuclear membrane.

\section{Materials and methods}

\section{Plasmid construction and mutagenesis}

All cloning procedures were performed according to standard methods [40]. cDNA encoding lamin A, cloned into the XhoI and EcoRI restriction site of the pCR 2.1TOPO vector (Invitrogen, Groningen, NL), was excised by restriction endonuclease digestion with XhoI and EcoRI and ligated into the $\mathrm{pCS} 2+$ vector and digested with the same restriction enzymes, pCS2 + vector is a pBluescript II KS+ -based plasmid [41] containing a FLAG-tag coding sequence inserted between the BamHI and EcoRI restriction sites by K. Köbernick (Department of Developmental Biochemistry, University of Göttingen, Germany). Point mutations were introduced into the full-length lamin cDNA using the QuikChange site-directed mutagenesis kit (Stratagene, La Jolla, CA).

\section{Cell culture and transfection}

HeLa SS6 cells were grown at $37^{\circ} \mathrm{C}$ with $5 \% \mathrm{CO}_{2}$ in Dulbecco's modified Eagle's medium supplemented with 
$10 \%$ fetal calf serum, penicillin, and streptomycin. Cells were regularly passaged to maintain exponential growth. The day before transfection, cells were trypsinized, diluted with fresh medium without antibiotics, and transferred to 35-mm dishes. Transient transfection of plasmid DNA was carried out by using Polyfect transfection reagent exactly as described by the manufacturer (Qiagen, Hilden, Germany). Cells were fixed and processed for immunofluorescence microscopy between 24 and $100 \mathrm{~h}$ post transfection. Half the medium was changed 3 days post transfection.

Cells that were trypsinized and replated were incubated for $2.5 \mathrm{~min}$ with TE buffer [0.1\% trypsin, $0.5 \mathrm{mM}$ EDTA in phosphate-buffered saline (PBS), pH 7.8], carefully resuspended, centrifuged (4 min, $1000 \mathrm{rpm}$ ), washed, and replated on coverslips marked with a grid (CELLocate, Eppendorf, Hamburg, Germany) at dilutions varying between $1: 20$ and $1: 160$.

\section{Immunofluorescence microscopy}

Cells grown on glass coverslips were fixed in methanol for $6 \mathrm{~min}$ at $-10^{\circ} \mathrm{C}$. Following (PBS) washes, the fixed cells were incubated with the first antibody for $30 \mathrm{~min}$ at $37^{\circ} \mathrm{C}$, washed with PBS, incubated with the second antibody for $30 \mathrm{~min}$ at $37^{\circ} \mathrm{C}$, again washed with PBS, and mounted in Mowiol 4-88 (Hoechst, Frankfurt, Germany).

The primary antibodies were monoclonal lamin A, JOL4 (Abcam, Cambridge, UK), monoclonal lamin B1, clone L-5 (Zymed Inc., San Francisco, CA), monoclonal lamin B2, clone LN43.2 (Novocastra, Newcastle, UK), polyclonal lamin C (Christopher Hutchison, University of Durham, UK), monoclonal emerin, clone 4G5 (Novocastra), monoclonal anti-FLAG M2 (Sigma-Aldrich, Deisenhofen, Germany), polyclonal anti-FLAG (Sigma-Aldrich), monoclonal LAP2, clone 27 (Transduction Laboratories, Lexington, KY), and monoclonal Nup153 (Brian Burke, University of Gainesville, FL). Appropriate dilutions of all antibodies were established by immunofluorescence microscopy.

Second antibodies obtained from Dianova (Hamburg, Germany) were fluorescein isothiocyanate-conjugated goat anti-mouse IgGs, rhodamine-conjugated goat anti-mouse IgGs, and rhodamine-conjugated goat anti-rabbit IgGs.

A Zeiss Axiophot with an F Fluar $40 \times / 1.30$ oil objective was used to collect digital images, which were further processed with MetaMorph Imaging Software (Universal Imaging Corporation, West Chester, PA).

\section{Transmission electron microscopy}

Cells on 12-mm glass coverslips were fixed in glutaraldehyde ( $2 \%$ in $0.1 \mathrm{M}$ cacodylate, $\mathrm{pH} 7.2,0.1 \mathrm{M}$ sucrose) for $45 \mathrm{~min}$ at room temperature. They were rinsed several times with $0.1 \mathrm{M}$ cacodylate, $\mathrm{pH} 7$, containing $0.1 \mathrm{M}$ sucrose and then briefly in distilled water. Following fixation with osmium tetroxide (2\% in $0.1 \mathrm{M}$ cacodylate, $\mathrm{pH} 7.2,0.1 \mathrm{M}$ sucrose), cells were stained en bloc with uranyl acetate ( $1 \%$ in water) overnight at $4^{\circ} \mathrm{C}$ in the dark. The cells were dehydrated in a graded series of ethanol and propylene oxide and flat-embedded in Epon. After polymerization of the Epon the coverslips were removed by etching in $48 \%$ hydrofluoric acid for $20 \mathrm{~min}$ at room temperature. They were rinsed three times in distilled water and dried overnight at $60^{\circ} \mathrm{C}$. The flat-embedded cells were sectioned serially parallel to the substratum. Sections were stained with uranyl acetate and lead citrate and examined in a Philips CM12 transmission electron microscope. Sections were approximately $90-100 \mathrm{~nm}$ thick and nuclear aggregates were most abundant between sections 25 and 35 .

\section{Immunoblotting}

Monoclonal 636 lamin A/C specific antibody [42] was used as hybridoma supernatant at a 1/100 dilution. For Western blotting, transfected cells grown in 2-ml dishes were trypsinized 3 days post transfection and harvested in Sodium dodecyl sulfate sample buffer (Bio-Rad Laboratories, Hercules, CA). Equal amounts of extract were separated on different lanes of a $12.5 \%$ polyacrylamide gels and transferred to nitrocellulose. Immunostaining was carried out by using the Renaissance Western Blot Chemiluminescence Reagent (NEN, Boston, MA) and analyzed by using the Lumi-Imager (Boehringer Mannheim, Mannheim, Germany) equipped with LumiAnalyst Version 3.0 software. The blots were then stripped by using the Western Blot Recycling kit (Chemicon International, Temecula, CA) and reprobed with the V9 vimentin antibody [43].

\section{Statistical methods}

Results are expressed as means \pm the standard deviation. Statistical analyses of aggregate size were performed with the Student's $t$ test using Excel software.

\section{Results}

\section{Abnormal organization of lamin A and formation of nuclear aggregates}

HeLa cells were transiently transfected with plasmids containing wild-type and mutated forms of lamin A. A FLAG epitope was present at the N-terminus of each construct, and lamin A was detected by indirect immunofluorescence with FLAG antibody. Results are shown in Fig. 1A for the wild-type lamin A construct and for the three lamin A mutations, i.e., G465D, R482L, and R527P. Lamin A molecules with G465D [8] and R482L [7] mutations have been reported in patients with FPLD, while the R527P mutation has been reported in a patient with EDMD [3]. All three mutations lie in the carboxy-terminal tail region in a subdomain characterized by the atomic structure of an Iglike domain $[44,45]$. 

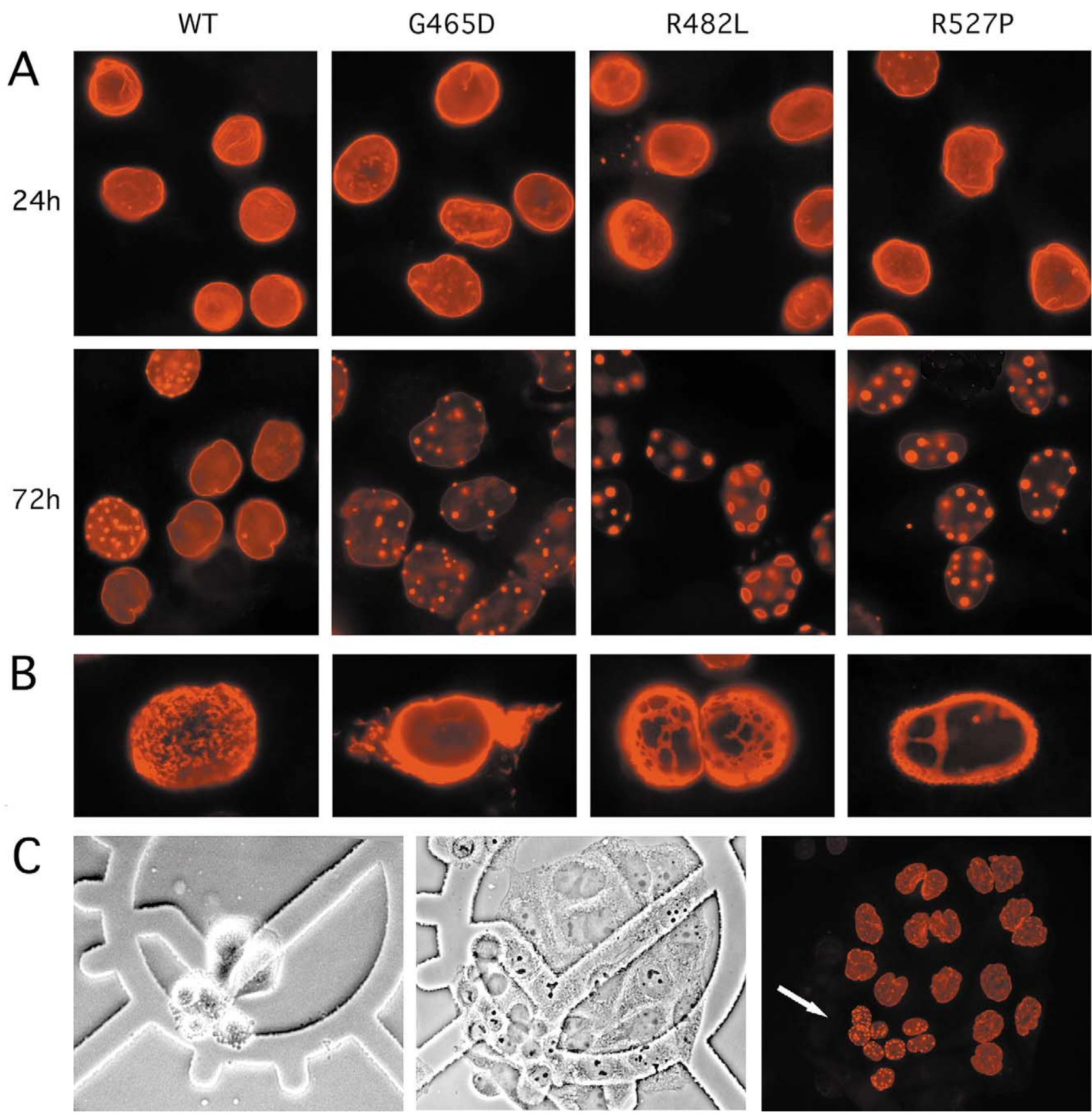

Fig. 1. (A) Nuclear localization of FLAG-tagged ectopically expressed wild-type and mutant forms of lamin A, 24 and $72 \mathrm{~h}$ post transfection. The panels show immunofluorescence microscopy images of HeLa SS6 cells transfected with wild-type lamin A (WT) or with the three lamin A missense mutants as indicated. Cells were stained with the mouse monoclonal antibody against FLAG and rhodamine-conjugated second antibody. Note the nuclear aggregates visible $72 \mathrm{~h}$ post transfection in cells transfected with all three mutant constructs as well as in wild-type transfected cells. (B) Abnormal arrangements of lamin A in individual HeLa cells transfected with the mutant G465D construct and stained with the monoclonal antibody against FLAG. (C) HeLa cells were trypsinized and replated onto marked coverslips $72 \mathrm{~h}$ post transfection with the R527P mutant construct. Individual colonies were followed in phase microscopy and photographed at $24 \mathrm{~h}$ (left) and at $48 \mathrm{~h}$ (not shown). At $72 \mathrm{~h}$ cells were fixed and stained for FLAG-tag to determine the lamin A location and photographed in phase (middle) and in fluorescence microscopy (right). The three micrographs show the same colony of cells. The presence of a group of eight cells with nuclear aggregates suggests that cells with aggregates can replicate (arrow). Magnifications: (A) $\times 660$, (B) $\times 1359$, and $(\mathrm{C}) \times 584$.

The data summarized in Fig. 1A show results at 24 and $72 \mathrm{~h}$ post transfection. At $24 \mathrm{~h}$ increased amounts of lamin A are present in both wild-type and mutant lamin A transfected cells, but the arrangement in most cells is comparable to that seen in untransfected cells. Nuclear aggregates are seen in $1-2 \%$ of cells in the cultures transfected with the wild-type or with the G465D mutant construct. However, already at this time point $7 \%$ of cells transfected with the R527P mutant and $13 \%$ of cells transfected with the R482L mutant construct show such aggregates (Fig. 1A). At $72 \mathrm{~h}$ 


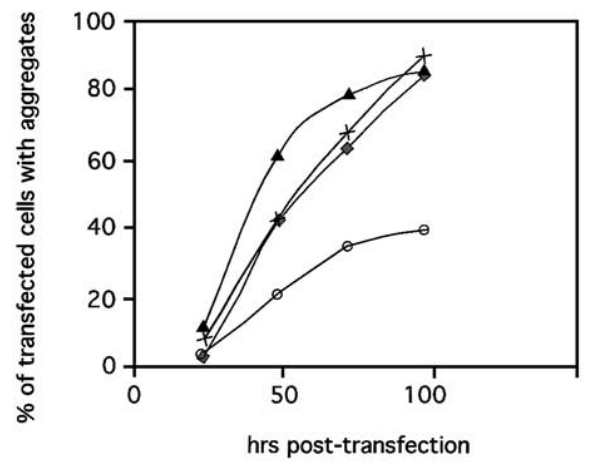

$\rightarrow$ G465D $\leadsto-\mathrm{R} 482 \mathrm{~L} \rightarrow \mathrm{R} 527 \mathrm{P}-\mathrm{WT}$

Fig. 2. The percentage of transfected cells in which nuclear aggregates can be visualized is plotted against time post transfection for HeLa cells transfected with each of the three mutant constructs and with wild-type cDNA. At least 200 transfected cells were counted for each point. Note the time-dependent increase of cells with aggregates, and that this increase is slower in wild-type than in mutant transfected cultures. Data shown are from a single experiment. Determination of the mean and standard deviation for three experiments gave the following values expressed as percentages of cells with aggregates $96 \mathrm{~h}$ post transfection: wild-type, $40.6 \pm$ $5.2 \%$; G465D, $85.4 \pm 1.2 \%$; R482L, $81.9 \pm 3.8 \%$; and R527P, $86.0 \pm$ $3.2 \%$. Interobserver variability in counting was $<5 \%$.

almost all of the FLAG-tagged lamin $\mathrm{A}$ is in the large nuclear aggregates in the three cultures transfected with the mutant constructs. However, such aggregates are also found to a lesser extent in cells transfected with the wild-type construct (Fig. 1A). Thus, at $72 \mathrm{~h}, 60-80 \%$ of the cells transfected with the mutant lamin constructs have aggregates when tested with the FLAG antibody, while at this time point only $\sim 35 \%$ of cells transfected with wild-type lamin A have aggregates. The kinetics of nuclear lamin aggregate formation is shown in Fig. 2. Here, the percentage of cells that contain nuclear aggregates has been plotted as a function of time after transfection. Transfected cells were followed for $96 \mathrm{~h}$ post transfection. At this time point $85-90 \%$ of cells transfected with the mutant cDNAs showed aggregates, while only some $40 \%$ of cells transfected with the wild-type cDNA had such aggregates (Fig. 2). Occasional metaphase cells with aggregates were also seen. Staining with Hoechst dye showed that DNA was excluded from the nuclear aggregates. Transfected cells were also tested with the TUNEL assay to determine the percentage of apoptotic cells. Only $2-5 \%$ were apoptotic at $48 \mathrm{~h}$ post transfection, and this number increased to $8-10 \%$ at $72 \mathrm{~h}$ post transfection.

The size of the nuclear aggregates was dependent on the time post transfection as well as on the particular mutant cDNA used to transfect the cells. Nuclear aggregates in the wild-type transfected cells were smaller than the aggregates in the mutants G465D and R527P, which in turn were smaller than those of the mutant R482L. Measurement of the aggregate diameters from immunofluorescence micrographs at $72 \mathrm{~h}$ post transfection yielded average values of
$0.83 \pm 0.22 \mu \mathrm{m}$ for the wild-type, $1.03 \pm 0.41 \mu \mathrm{m}$ for the mutant R527P, $1.02 \pm 0.31 \mu \mathrm{m}$ for the mutant G465D, and $1.54 \pm 0.54 \mu \mathrm{m}$ for the mutant R482L (Table 1). These differences in size in aggregate diameter are significant for wild-type vs. each of the three mutants and for R527P and G465D vs. R482L $(P<0.001)$. As far as the number of aggregates is concerned, cells transfected with the R482L mutant appeared to have fewer aggregates than cells transfected with the other two mutant cDNAs at the same time point (Table 1). Some FLAG-tagged lamin A was found distributed throughout the nucleoplasm and underlying the nuclear membrane, as well as in the nuclear aggregates. This location of FLAG-tagged lamin A was more pronounced in cells containing aggregates resulting from transfection with the wild-type cDNA (Fig. 1A, row 2).

Finally, in some transfected cells and particularly in those transfected with the mutant cDNAs, it was clear that overexpression of lamin A resulted in additional bizarre arrangements of lamin A. Lamin A was found in occasional cells in whirls, or in large aggregates subsuming the nuclear membrane, or in some instances even as aggregates in the cytoplasm (Fig. 1B). Thus, the percentages of cells with clearly aberrant lamin A arrangements are larger than those shown in Fig. 2, which includes only those cells with clearly identifiable nuclear aggregates.

Fibroblasts from patients with FPLD show aberrant nuclear morphologies after heat shock [37]. We therefore tested whether the lamin A distribution seen in our experiments was sensitive to heat shock $70 \mathrm{~h}$ post transfection. Cells transfected with wild-type or the mutant DNA constructs were incubated at $42^{\circ} \mathrm{C}$ for $1 \mathrm{~h}$ and then tested with the anti-FLAG antibody (data not shown). No change was seen either in the nuclear shape or in the aggregates, sug-

Table 1

Comparison of aggregate diameter in wild-type and mutant cultures by immunofluorescence (IF) and electron microscopy (EM) ${ }^{\mathrm{a}}$

\begin{tabular}{lcccc}
\hline & Wild-type & R527P & G465D & R482L \\
\hline IF & & & & \\
Mean & 0.83 & 1.03 & 1.02 & 1.54 \\
SD & 0.22 & 0.41 & 0.31 & 0.54 \\
$n$ & 141 & 89 & 53 & 35 \\
EM & & & & \\
Mean & 0.73 & 0.98 & 1.09 & 1.34 \\
SD & 0.22 & 0.33 & 0.44 & 0.54 \\
$n$ & 77 & 62 & 98 & 53 \\
\hline
\end{tabular}

${ }^{\mathrm{a}}$ Ten cells with aggregates were chosen at random from immunofluorescence (IF) and electronmicroscopy (EM) prints. The diameter of each aggregate was measured, and the mean and standard deviations calculated. Note the agreement between the values obtained for the aggregate diameters in immunofluorescence and in electron microscopy. Note that the aggregates in wild-type transfected cells were smaller than those in R527P and G465D transfected cells, which in turn were smaller than those in R482L transfected cells. (IF: wild-type vs. R527P, G465D, or R482L, $P<0.001$ : R527P or G465D vs. R482L, $P<0.001$; R527P vs. G465D, not significant. For EM the $P$ values are identical to those given for IF, except for G465D vs. R482L, where $P<0.005$.) SD, standard deviation; $n$, number of aggregates. 
gesting that under our conditions these structures are heat shock insensitive.

To test whether HeLa cells with nuclear aggregates can replicate, cells transfected with the R527P mutant construct were trypsinized and replated on to marked coverslips $72 \mathrm{~h}$ post transfection. Staining of a parallel coverslip showed that prior to trypsinization $77 \%$ of the cells were transfected and $39 \%$ of the transfected cells had aggregates. Several colonies on the marked coverslips were selected and photographed 24, 48, and $72 \mathrm{~h}$ after replating. At $72 \mathrm{~h}$ after replating (144 $\mathrm{h}$ post transfection) immunofluorescence with the FLAG-tagged lamin A antibody showed that $17 \%$ of the cells were transfected and that $27 \%$ of the transfected cells had aggregates. Thus, transfected cells, as well as cells with aggregates, survive replating although the percentage of transfected cells decreases with time after replating. As can be seen in Fig. 1C, the number of cells in an individual colony increased with time. In such colonies often both types of cells are visible, i.e., cells in which the FLAGtagged mutant lamin A shows a relatively normal distribution, as well as cells in which nuclear aggregates are clearly apparent. Micrographs of the type shown in Fig. 1C, in which clusters of cells apparently derived from a single cell have aggregates, are consistent with the possibility that cells with nuclear aggregates can replicate.

\section{Levels of expression of lamin A in transfected cells}

The data in Figs. 1A and 2 suggest strongly that formation of nuclear aggregates is caused at least in part by overexpression of lamin A in the transfected cells. For this reason we decided to measure the levels of overexpression in HeLa cultures transfected with either the wild-type lamin A or with the mutant lamin A constructs.

Extracts of untransfected (NT) and of wild-type (WT) or mutant transfected HeLa cells obtained $72 \mathrm{~h}$ post transfection were subjected to sodium dodecyl sulfate-electrophoresis and immunoblotting with the 636 lamin monoclonal antibody, which recognizes both lamin $\mathrm{A}$ and lamin $\mathrm{C}$ (Fig. 3). The results obtained when the blots are scanned with the Lumi-Imager are shown in panel A. The immunoblots were then stripped and reprobed with the V9 vimentin antibody (panel B). The extent of overexpression expressed either as real counts, or on a per-transfected-cell basis, is shown in panel C. Both overexpression ratios assign an arbitrary value of 1 to the untransfected culture grown in parallel. The results show that on a per-transfected-cell basis lamin $\mathrm{A}$ is overexpressed in the $\mathrm{R} 482 \mathrm{~L}$ culture by a factor of 10 , in the R527P culture by a factor of 20 , and in the G465D culture by a factor of 24 . In the wild-type culture lamin A is overexpressed by a factor of 20 . The transfection efficiency for the R482L construct in the experiment shown in Fig. 3 was relatively low (20\%) vs. those seen with the G465D (75\%) and R527P (70\%) and the wild-type construct $(60 \%)$. However, in additional experiments in which the $\mathrm{R} 482 \mathrm{~L}$ transfection rates were similar to wild-type,
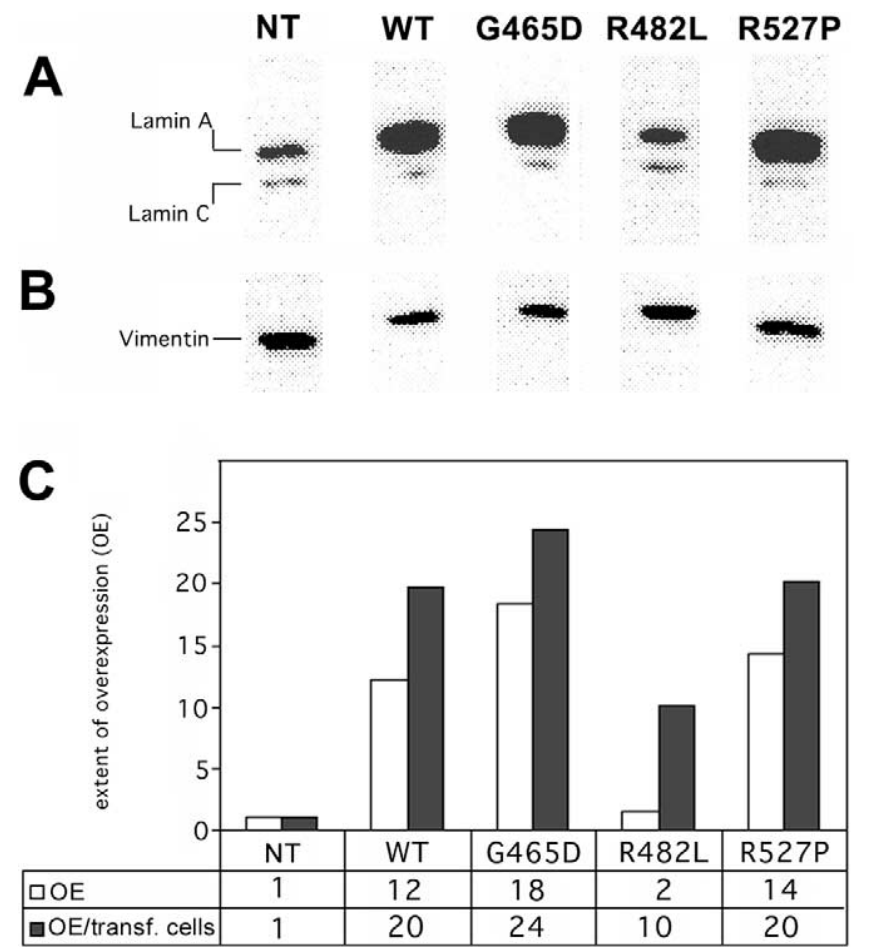

Fig. 3. (A) Untransfected (NT), wild-type (WT), or mutant transfected HeLa cells were harvested $72 \mathrm{~h}$ post transfection and separated on sodium dodecyl sulfate gels. Immunoblots of the extracts were assayed with the lamin A/C 636 monoclonal antibody and read on the Lumi-Imager. Equal amounts of extract were loaded. (B) The immunoblots were stripped and reprobed with the V9 vimentin monoclonal antibody. (C) The unfilled bars show the ratio of counts for lamin A for the cultures in A normalized to the nontransfected culture arbitrarily assigned a value of 1 . The black bars show the extent of overexpression (OE) calculated on a per-transfected-cell basis, again with the nontransfected culture assigned a value of 1 . The WT cultures express approximately twice as much lamin A as the R482L culture and about the same amount as the R527P culture on a per-transfected-cell basis. Note that the R482L culture expresses less lamin A on a per-cell basis than do the cultures transfected with wild-type or with the G465D and R527P mutants. The reduced aggregate formation seen with the WT culture cannot therefore be explained by lower levels of lamin A overexpression in the WT culture.

overexpression/cell ratios similar to those shown in Fig. 3 were measured for R482L and for wild-type (data not shown).

Thus, the combined results show that the lower rate of nuclear aggregate formation seen with the wild-type vs. the mutants is not a result of lower expression. The wild-type cells express twice as much lamin A as the R482L-transfected cultures and the same amount as the R527P cultures on a per-transfected-cell basis.

\section{Other lamins and lamina-associated proteins are recruited into the aggregates}

We next examined the location of proteins known to be associated with the nuclear lamina in cells with nuclear aggregates. Results are shown in Fig. 4 for HeLa cells 
transfected with the R527P lamin A mutant construct for lamin C, lamin B1, lamin B2, LAP2, Nup153, and emerin $48 \mathrm{~h}$ post transfection. Examination of the individual panels of Fig. 4 as well as of the overlays shows a colocalization to the nuclear aggregates for five of the six proteins. The endogenous lamin $\mathrm{C}$ is recruited into the aggregates when probed in double label with a lamin C-specific antibody. Little or no staining of the nuclear lamina is detected with the lamin $\mathrm{C}$ antibody in the cells with aggregates (Fig. 4, top row). Cells with aggregates stained with the monoclonal lamin A antibody also showed little staining of the nuclear lamina, but strong staining of the aggregates (data not shown). Lamin B1, lamin B2, LAP2, and Nup153 also are found in nuclear aggregates (Fig. 4, rows 2-5), but in contrast staining of the nuclear lamina is seen with antibodies to these proteins (Fig. 4, column 1). The sixth protein, emerin, is found associated with the nuclear lamina in cells without aggregates. A normal emerin stain with no staining of the aggregates is seen in 55\% of cells with aggregates. Staining of the lamina, as well as an association with nuclear aggregates, is seen in approximately $40 \%$ of cells showing such aggregates. It was usually stronger at the periphery of the aggregates than in the center (Fig. 4, row $6)$. In very few cells in the culture $(<2 \%)$ a noncolocalization of emerin with the FLAG-tagged lamin A was observed. In such cells no staining of aggregates was seen with the emerin antibody and staining of the nuclear lamina was reduced. Instead emerin was detected in the cytoplasm as small aggregates that may correspond to strands of the endoplasmic reticulum, while lamin A was found in nuclear aggregates (Fig. 4, row 7). In a further $2-3 \%$ of cells a complete loss of emerin staining was observed. Overexpression of the R527P lamin A mutant cDNA thus causes an aberrant localization and a redistribution of many of the proteins normally associated with the nuclear envelope to the nuclear aggregates.

Location of lamina proteins was also studied in HeLa cells transfected with the mutants G465D and R527P as well as with wild-type at 4 days post transfection. There was only a single difference to the results presented in Fig. 4. At 4 days post transfection, emerin did not appear to colocalize with nuclear aggregates in cells transfected with the wildtype construct.

\section{Electron microscopy of nuclear aggregates in lamin A- transfected cells}

HeLa cells transfected with the three mutant lamin A cDNA constructs and with the wild-type cDNA construct were subjected to electron microscopy at $73 \mathrm{~h}$ post transfection. This time point was chosen to ensure that it would be relatively easy to find cells in which nuclear aggregates were present.

Examination of Fig. 5 shows that it is possible to obtain electron micrographs in which large numbers of nuclear aggregates are present in cells transfected with each of the lamin A mutant constructs (Fig. 5A-C) and with the wildtype lamin A construct (Fig. 5D). Measurement of the aggregate diameter at $72 \mathrm{~h}$ post transfection yielded average values of $0.73 \pm 0.22 \mu \mathrm{m}$ for the wild-type, $0.98 \pm 0.33$ $\mu \mathrm{m}$ for the mutant R527P, $1.09 \pm 0.44 \mu \mathrm{m}$ for the mutant $\mathrm{G} 465 \mathrm{D}$, and $1.34 \pm 0.54 \mu \mathrm{m}$ for the R482L mutant. These differences in size are significant for wild-type vs. R527P and G465D and R482L and for R527P and G465D vs. $\mathrm{R} 482 \mathrm{~L}$ (legend to Table 1). The size differences are also apparent in Fig. 5 where panels A-D are shown at the same magnification. Aggregates are sometimes found within the nucleus, apparently unassociated with the nuclear membrane (Fig. 5A and C) although occasionally (insert in Fig. 5C) a membrane fragment can be found attached to an individual aggregate. However, by far, the majority of the aggregates at this time point are found associated with and often underlying the nuclear membrane (e.g., Fig. 5B). In some cells aggregates are found that seem to push through the nuclear membrane (e.g., Fig. 5C). In this process they appear to become surrounded by a membrane. In a few cells in which aggregates are clearly present in the cytoplasm such aggregates also appear surrounded by a membrane. No substructure was visible in the aggregates at higher resolution.

\section{Discussion}

Nuclear aggregates result in part from overexpression and in part from mutant-specific effects

Our results show that all three mutations examined in our study form nuclear aggregates, detectable both in immunofluorescence and in electron microscopy, and that the number of such aggregates increased with time after transfection (Fig. 2). Aggregate formation increases with time until at $96 \mathrm{~h}$ more than $80 \%$ of the transfected cells have aggregates. New in our study is the observation that cells transfected with the wild-type lamin A construct also show the nuclear aggregates at later times, albeit to a lesser extent than the three mutant lamin A constructs that we studied. Thus, at $96 \mathrm{~h}$ around $40 \%$ of cells transfected with the wild-type construct have aggregates, i.e., about half the level seen with the mutant transfected cells at the same time point.

The finding of aggregates in the wild-type transfected cells, as well as the increase in nuclear aggregate formation with time, suggests that aggregates may be formed in part as a consequence of overexpression of mutant or wild-type lamin A. Fig. 3 shows that mutant transfected cells overexpress lamin A by a factor of 10-24, and wild-type by a factor of 20, when compared to the untransfected cell level. Two further arguments suggest a possible link between overexpression and aggregate formation. First, primary cultures of skin fibroblasts from patients with FPLD containing the R482Q or R482W mutations show no aggregate formation. These cells express normal amounts of the mutant 


\section{Specific ab}

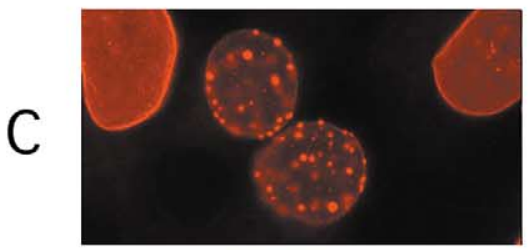

B1

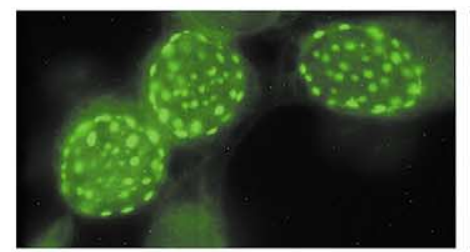

B2

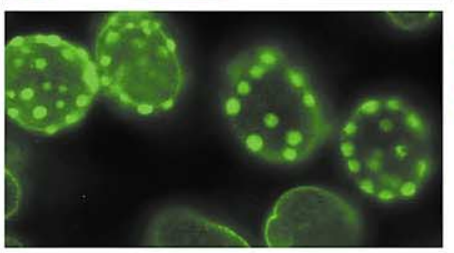

LAP 2

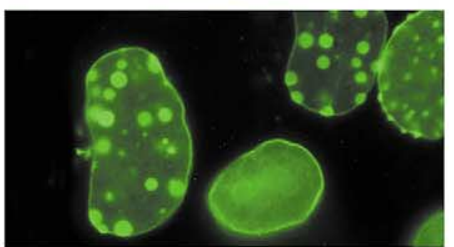

Nup153
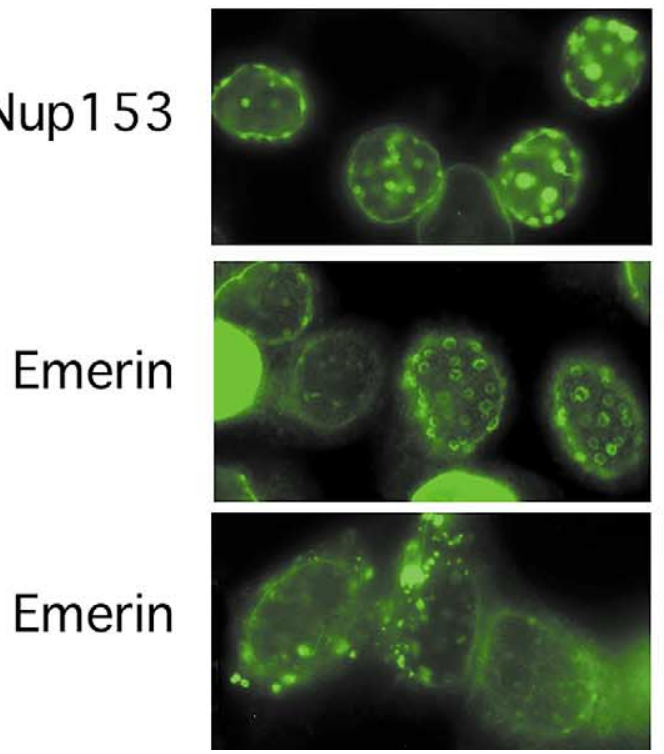

Anti-FLAG
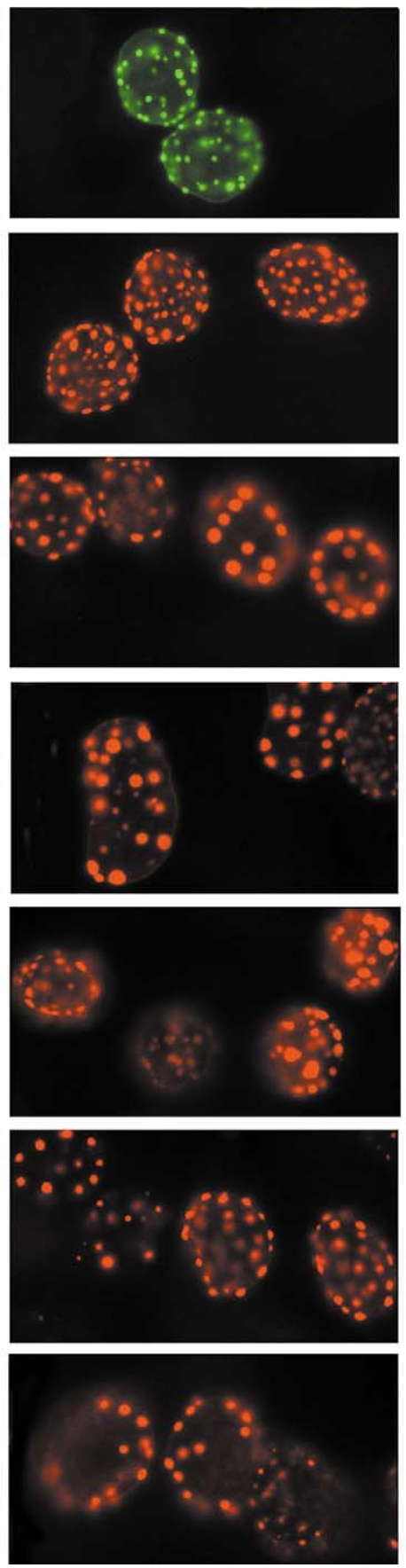

Overlay
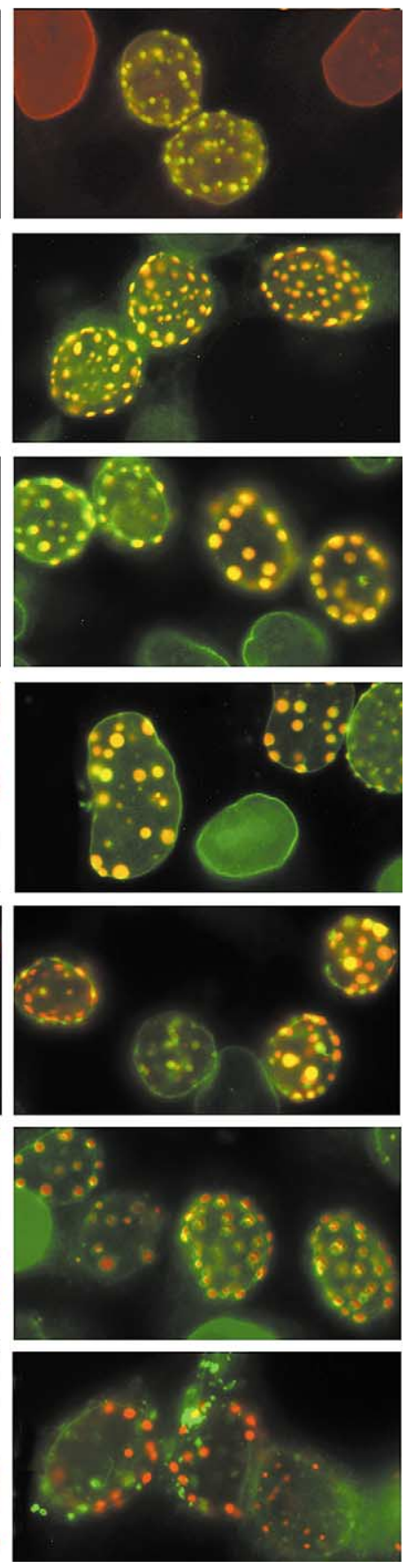

Fig. 4. Double immunofluorescence analysis of HeLa cells expressing the R527P mutant form of lamin A $48 \mathrm{~h}$ post transfection. For lamin $\mathrm{C}$ a mouse anti-FLAG antibody and a rabbit lamin C antibody were used. For the other proteins a rabbit anti-FLAG antibody was used to reveal the FLAG-tagged mutant lamin (middle panels) and a mouse antibody specific for each of the endogenous proteins (left panels). The aberrant localization of the mutated lamin A causes the endogenous proteins to delocalize and become incorporated into the nuclear aggregates (see overlays in the third column). In all cases except lamin $\mathrm{C}$, considerable staining of the nuclear lamina is also observed. With emerin antibody a rim staining of the intracellular aggregates is seen in approximately half the cells with aggregates (row 6). In a few cells $(<2 \%)$ emerin localizes not to the nuclear aggregates but to strands in the cytoplasm (row 7$)$ (see text). Magnification: $\times 808$.

lamin A [37]. Second, aggregate formation was detected in one study for mutant lamin B1 that resulted in a two- to four-fold overexpression of lamin B1 [46], while it was not seen in a study of mutant lamin B2 in which expression of the mutant lamin was two- to four-fold less than of the endogenous lamin [47].

Given our results that aggregate formation is dependent on the time after transfection at which cells are examined, as 

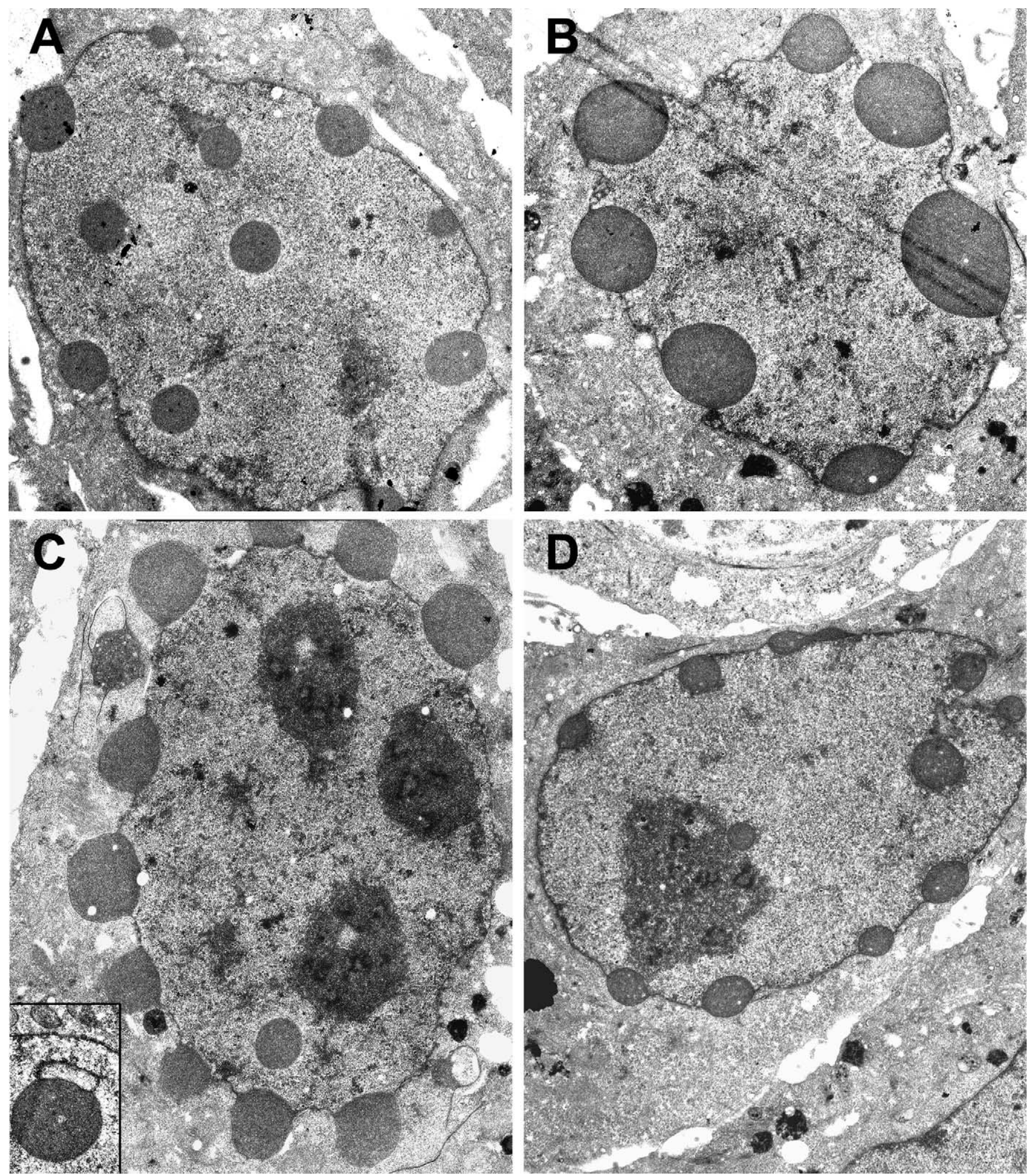

Fig. 5. Electron microscopy of HeLa cells transfected with mutant R527P (A); mutant R482L (B and inset in C); mutant G465D (C); and wild-type construct (D). Note that many aggregates are found associated with the nuclear membrane and that aggregates are also found inside the nucleus (A, C, and D). Note the association of the aggregate inside the nucleus with a fragment of nuclear membrane (inset in C). Magnifications: $(A-D) \times 8800$ and (inset in C) $\times 10,000$.

well as on the amount of lamin A synthesized, it is possible to resolve discrepancies apparent in previous studies of cells transfected with lamin A mutant constructs. Thus, in one study [38] only a single mutant (N195K) was reported to form aggregates when tested $20-24 \mathrm{~h}$ post transfection. In a second study [39] transfection with four mutant lamin A 
constructs (N195K, E358K, M371K, and R386K) resulted in nuclear aggregates at $24 \mathrm{~h}$ post transfection. Eleven other mutant lamin A constructs, including three that involve changes at two of the same amino acid positions as used in this study (R482Q, R482W, and R527P), did not form aggregates at $24 \mathrm{~h}$, the only time point that was tested. Our data on all three mutants, and in particular on the R482L and on the R527P mutant, show aggregate formation in a low

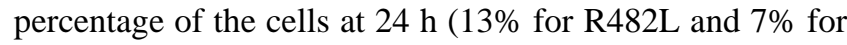
R527P). Aggregate formation increases with time until after $100 \mathrm{~h}$ 85-90\% of cells transfected with the mutant cDNA constructs have aggregates.

Overexpression alone, however, seems insufficient to explain the quantitative difference between wild-type and mutant cDNAs, since wild-type transfected cells express about twice as much lamin A as the R482L mutant and about the same as the R527P mutant. The data thus suggest that there is an additional effect due to the specific mutations in the lamin A gene. A further argument in support of mutant-specific effects is the different sizes of the aggregates seen with the different mutants and with the wild-type in both immunofluorescence and electron microscopy (Figs. 1A and 5). Values for aggregate diameters from the immunofluorescence micrographs and from the electron micrographs agree well with each other. Our data show that aggregates of wild-type lamin A are on average smaller than those seen with the G465D and R527P mutant lamins, and these in turn are smaller than those seen with the R482L mutation.

However, mutants causing a particular disease could not be placed into groups by looking at features of the aggregates. For example, G465D, a mutation characteristic of patients with FPLD, and R527P, a mutation characteristic of patients with EDMD, appeared to form very similar aggregates at both the light and electron microscopic levels. The R482L mutant (FPLD) formed aggregates that were larger than those seen in the other two mutants both by light and electron microscopy.

\section{Effect of mutations on nuclear stability}

Abnormal nuclear envelope herniations have been detected in fibroblasts from patients with FPLD subjected to heat shock [37]. No such effects were seen when the lamin A mutants used in this study were subjected to the same conditions. Instead the nuclei retained their usual shape even though most of the lamin A and lamin C, as well as the other lamins and lamin-associated proteins, were recruited into the aggregates. Lamin A was not extractable by Triton $\mathrm{X}-100$ as judged by immunofluorescence microscopy of cells transfected with the mutants or with the wild-type [46].

\section{Influence of aggregates on other lamins and on lamin- associated proteins}

Lamins C, B1, and B2 are recruited into the aggregates. In cells with aggregates little staining of the nuclear lamina is seen with the lamin C-specific antibodies, but the lamina is more strongly stained with the B1 and B2 antibodies. The lamin-associated proteins LAP2 and emerin were also recruited into aggregates, and these proteins are also found in the nuclear lamina in cells with aggregates. Also, the nuclear pore protein Nup153 is found associated with the aggregates (Fig. 4).

Emerin staining was detected as a ring-like stain around some but not all aggregates. For the R527P mutant no change was seen in the level of emerin under the nuclear membrane when cells with aggregates were examined. This mutation, therefore, like the R482W/Q mutations studied at $24 \mathrm{~h}$ by Östlund et al. [39], appears not to exert a dominant negative effect on emerin localization at the nuclear membrane. This is in contrast to a variety of other lamin A mutations studied by others $[38,39]$ in which up to $50 \%$ of the cells show a loss of nuclear envelope-associated emerin. In addition, cell type-specific alterations in emerin distribution and nuclear envelope fragility have been reported in mice lacking the A-type lamins [24]. Staining of nuclear aggregates with emerin antibodies has not always been seen in previous studies [38,39], and such staining may therefore be associated with particular mutations in the lamin A gene and/or with late times post transfection.

It remains an intriguing question how different mutations in a single gene can result in six different diseases. Three mechanisms have been suggested $[13,29,48]$. First that nuclei in cells with lamin A mutations are more fragile, second that the nuclear envelope may play a significant role in regulating gene expression patterns, and third that there could be a knock-on effect dependent on the complement of proteins found in the endoplasmic reticulum. Currently neither we or others can decide between these mechanisms, nor is it clear whether a single mechanism can explain all six laminopathies. Nevertheless, our data suggest that in tissue culture the answer is most likely to come from studies such as that of Vigouroux et al. [37] in which the level of lamin A expression within the cells is not perturbed. This is perhaps particularly important insofar as four of the six diseases result from heterozygous mutations in the lamin A gene.

\section{Acknowledgments}

We thank Heinz-Jürgen Dehne and Susanne Brandfass for expert technical assistance, Heinz-Jürgen Dehne for help with the electron microscopy, and Drs. Brian Burke and Christopher Hutchison for supplying the Nup153 and the lamin $\mathrm{C}$ antibodies, respectively.

\section{References}

[1] D. Fatkin, C. MacRae, T. Sasaki, M.R. Wolff, M. Porcu, M. Frenneaux, J. Atherton, H.J. Vidaillet, S. Spudich, U. De Girolami, J.G. 
Seidman, C.E. Seidman, Missense mutations in the rod domain of the lamin $\mathrm{A} / \mathrm{C}$ gene as causes of dilated cardiomyopathy and conductionsystem disease, N. Engl. J. Med. 341 (1999) 1715-1724.

[2] A. Muchir, G. Bonne, A.J. van der Kooi, M. van Meegen, F. Baas, P.A. Bolhuis, M. de Visser, K. Schwartz, Identification of mutations in the gene encoding lamins $\mathrm{A} / \mathrm{C}$ in autosomal dominant limb girdle muscular dystrophy with atrioventricular conduction disturbances (LGMD1B), Hum. Mol. Genet. 9 (2000) 1453-1459.

[3] G. Bonne, M.R. Di Barletta, S. Varnous, H.-M. Bécane, E.-H. Hammouda, L. Merlini, F. Muntoni, C.R. Greenberg, F. Gary, J.-A, Duboc, D.M. Urtizberea Fardeau, D. Toniolo, K. Schwartz, Mutations in the gene encoding lamin $\mathrm{A} / \mathrm{C}$ cause autosomal dominant Emery-Dreifuss muscular dystrophy, Nat. Genet. 21 (1999) 285-288.

[4] M.R. Di Barletta, E. Ricci, G. Galluzzi, P. Tonali, M. Mora, L. Morandi, A. Romorini, T. Voit, K.H. Orstavik, L. Merlini, C. Trevisan, V. Biancalana, I. Housmanowa-Petrusewicz, S. Bione, R. Ricotti, K. Schwartz, G. Bonne, D. Toniolo, Different mutations in the LMNA gene cause autosomal dominant and autosomal recessive Emery-Dreifuss muscular dystrophy, Am. J. Hum. Genet. 66 (2000) 1407-1412.

[5] A.E.H. Emery, F.E. Dreifuss, Unusual type of benign X-linked muscular dystrophy, J. Neurol. Neurosurg. Psychiatry 29 (1966) 338342.

[6] H. Cao, R.A. Hegele, Nuclear lamin A/C R482Q mutation in Canadian kindreds with Dunnigan-type familial partial lipodystrophy, Hum. Mol. Genet. 9 (2000) 109-112.

[7] S. Shackleton, D.J. Lloyd, S.N. Jackson, R. Evans, M.F. Niermeijer, B.M. Singh, H. Schmidt, G. Brabant, S. Kumar, P.N. Durrington, S. Gregory, S. O'Rahilly, R.C. Trembath, LMNA, encoding lamin A/C, is mutated in partial lipodystrophy, Nat. Genet. 24 (2000) 153-156.

[8] R.A. Speckman, A. Garg, F. Du, L. Bennet, R. Veile, E. Arioglu, S.I. Taylor, M. Lovett, A.M. Bowcock, Mutational and haplotype analyses of families with familial partial lipodystrophy (Dunnigan variety) reveal recurrent missense mutations in the globular C-terminal domain of lamin A/C, Am. J. Hum. Genet. 66 (2000) 1192-1198.

[9] J. Köbberling, M. Dunnigan, Familial partial lipodystrophy: two types of an X-linked dominant syndrome, lethal in the hemizygous state, J. Med. Genet. 23 (1986) 120-127.

[10] A. De Sandre-Giovannoli, M. Chaouch, S. Kozlov, J.M. Vallat, M. Tazir, N. Kassouri, P. Szepetowski, T. Hammadouche, A. Vandenberghe, C.L. Stewart, D. Grid, N. Levy, Homozygous defects in $L M N A$, encoding lamin A/C nuclear-envelope proteins, cause autosomal recessive axonal neuropathy in human (Charcot-Marie-Tooth disorder type 2) and mouse, Am. J. Hum. Genet. 70 (2002) 726-736.

[11] G. Novelli, A. Muchir, F. Sangiuolo, A. Helbling-Leclerc, M.R. D'Apice, C. Massart, F. Capon, P. Sbraccia, M. Federici, R. Lauro, C. Tudisco, R. Pallotta, G. Scarano, B. Dallapiccola, L. Merlini, G. Bonne, Mandibuloacral dysplasia is caused by a mutation in LMNAencoding lamin A/C, Am. J. Hum. Genet. 71 (2002) 426-431.

[12] J. Genschel, H.H.J. Schmidt, Mutations in the LMNA gene encoding lamin A/C, Hum. Mutat. 16 (2000) 451-459.

[13] C.J. Hutchison, M. Alvarez-Reyes, O.A. Vaughan, Lamins in disease: why do ubiquitously expressed nuclear envelope proteins give rise to tissue-specific disease phenotypes?, J. Cell Sci. 114 (2001) 9-19.

[14] D.Z. Fisher, N. Chaudhary, G. Blobel, cDNA sequencing of nuclear lamins $\mathrm{A}$ and $\mathrm{C}$ reveals primary and secondary structural homology to intermediate filament proteins, Proc. Natl. Acad. Sci. USA 83 (1986) $6450-6454$.

[15] F. Lin, H.J. Worman, Structural organization of the human gene encoding nuclear lamin A and nuclear lamin C.J, Biol. Chem. 268 (1993) 16321-16326.

[16] B.M. Machiels, A.H.G. Zorenc, J.M. Endert, H.J.H. Kuijpers, G.J.J.M. van Eys, F.C.S. Ramaekers, J.L.V. Broers, An alternative splicing product of the lamin A/C gene lacks exon 10, J. Biol. Chem. 271 (1996) 9249-9253.
[17] C. Stewart, B. Burke, Teratocarcinoma stem cells and early mouse embryos contain only a single major lamin polypeptide closely resembling lamin B, Cell 51 (1987) 383-392.

[18] R.-A. Röber,, K. Weber, M. Osborn, Differential timing of nuclear lamin $\mathrm{A} / \mathrm{C}$ expression in the various organs of the mouse embryo and the young animal: a developmental study, Development 105 (1989) 365-378.

[19] R.A. Röber, H. Sauter, K. Weber, M. Osborn, Cells of the cellular immune and hemopoietic system of the mouse lack lamins $\mathrm{A} / \mathrm{C}$ : distinction versus other somatic cells, J. Cell Sci. 95 (1990) 587-598.

[20] L. Gerace, B. Burke, Functional organization of the nuclear envelope, Annu. Rev. Cell Biol. 4 (1988) 335-374.

[21] N. Stuurman, S. Heins, U. Aebi, Nuclear lamins: their structure, assembly and interactions, J. Struct. Biol. 122 (1998) 42-66.

[22] H.J. Worman, J.-C. Courvalin, The inner nuclear membrane, J. Membr. Biol. 177 (2000) 1-11.

[23] K. Furukawa, Y. Hotta, cDNA cloning of a germ cell-specific lamin B3 from mouse spermatocytes and analysis of its ectopic expression in somatic cells, EMBO J. 12 (1993) 97-106.

[24] T. Sullivan, D. Escalante-Alcalde, H. Bhatt, M. Anver, N. Bhat, K. Nagashima, C.L. Stewart, B. Burke, Loss of A-type lamin expression compromises nuclear envelope integrity leading to muscular dystrophy, J. Cell Biol. 147 (1999) 913-919.

[25] J. Harborth, S.M. Elbashir, K. Bechert, T. Tuschl, K. Weber, Identification of essential genes in cultured mammalian cells using small interfering RNAs, J. Cell Sci. 114 (2001) 4557-4565.

[26] L. Gerace, A. Blum, G. Blobel, Immunocytochemical localization of the major polypeptides of the nuclear pore complex-lamina fraction: interphase and mitotic distribution, J. Cell Biol. 79 (1978) 546-566.

[27] U. Aebi, J. Cohn, L. Buhle, L. Gerace, The nuclear lamina is a meshwork of intermediate-type filaments, Nature 323 (1986) 560564.

[28] M. Cohen, K.K. Lee, K.L. Wilson, Y. Gruenbaum, Transcriptional repression, apoptosis, human disease and the functional evolution of the nuclear lamina, Trends Biochem. Sci. 26 (2001) 41-47.

[29] B. Burke, L.C. Mounkes, C.L. Stewart, The nuclear envelope in muscular dystrophy and cardiovascular diseases, Traffic 2 (2001) 675-683.

[30] E.A. Fairley, J. Kendrick-Jones, J.A. Ellis, The Emery-Dreifuss muscular dystrophy phenotype arises from aberrant targeting and binding of emerin at the inner nuclear membrane, J. Cell Sci. 112 (1999) 2571-2582.

[31] L. Clements, S. Manilal, D.R. Love, G.E. Morris, Direct interaction between emerin and lamin A, Biochem. Biophys. Res. Commun. 267 (2000) 709-714.

[32] R. Foisner, L. Gerace, Integral membrane proteins of the nuclear envelope interact with lamins and chromosomes, and binding is modulated by mitotic phosphorylation, Cell 73 (1993) 1267-1279.

[33] T. Dechat, B. Korbei, S.V. Vaughan, S. Vlcek, C.J. Hutchison, R. Foisner, Lamina-associated polypeptide $2 \alpha$ binds intranuclear A-type lamins, J. Cell. Sci. 113 (2000) 3473-3484.

[34] H.J. Worman, J. Yuan, G. Blobel, S.D. Georgatos, A lamin B receptor in the nuclear envelope, Proc. Natl. Acad. Sci. USA 85 (1988) 8531-8534.

[35] C. Smythe, H.E. Jenkins, J. Hutchison, Incorporation of the nuclear pore basket protein Nup153 into nuclear pore structure is dependent upon lamina assembly: evidence from cell-free extracts of Xenopus eggs, EMBO. J. 19 (2000) 3918-3931.

[36] H. Taniura, C. Glass, L. Gerace, A chromatin binding site in the tail domain of nuclear lamins that interacts with core histones, J. Cell Biol. 131 (1995) 33-44.

[37] C. Vigouroux, M. Auclair, E. Dubosclard, M. Pouchelet, S. Lepanse, J. Capeau, J.-C. Courvalin, B. Buendia, Nuclear envelope disorganization in fibroblasts from lipodystrophic patients with heterozygous R482Q/W mutations in lamin A/C gene, J. Cell Sci. 114 (2001) $4459-4468$. 
[38] W.H. Raharjo, P. Enarson, T. Sullivan, C. Stewart, B. Burke, Nuclear envelope defects associated with LMNA mutations causing dilated cardiomyopathy and Emery-Dreifuss muscular dystrophy, J. Cell Sci. 114 (2001) 4447-4457.

[39] C. Östlund, G. Bonne, K. Schwartz, H. Worman, Properties of lamin A found in Emery-Dreifuss muscular dystrophy, cardiomyopathy and Dunnigan-type partial lipodystrophy, J. Cell Sci. 114 (2001) 44354445.

[40] J. Sambrook, E.F. Fritsch, T. Maniatis, Molecular Cloning: A Laboratory Manual, Cold Spring Harbor Laboratory Press, Cold Spring Harbor, NY, 1994.

[41] R.A.W. Rupp, L. Snider, H. Weintraub, Xenopus embryos regulate the nuclear localization of XMyoD, Genes Dev. 8 (1994) 13111323.

[42] R.A. Röber, R.K.H. Gieseler, J.H. Peters, K. Weber, M. Osborn, Induction of nuclear lamins $\mathrm{A} / \mathrm{C}$ in macrophages in in vitro cultures of rat bone marrow precursor cells and human blood monocytes, and in macrophages elicited in vivo by thioglycollate stimulation, Exp. Cell Res. 190 (1990) 185-194.
[43] M. Osborn, E. Debus, K. Weber, Monoclonal antibodies specific for vimentin, Eur. J. Cell Biol. 34 (1984) 137-143.

[44] I. Krimm, C. Östlund, B. Gilquin, J. Couprie, P. Hossenlopp, J.P. Mornon, G. Bonne, J.C. Courvalin, H.J. Worman, S. Zinn-Justin, The Ig-like structure of the $\mathrm{C}$-terminal domain of lamin $\mathrm{A} / \mathrm{C}$, mutated in muscular dystrophies, cardiomyopathy, and partial lipodystrophy, Structure 10 (2002) 813-823.

[45] S. Dhe-Paganon, E.D. Werner, Y.I. Chi, S.E. Shoelson, Structure of the globular tail of nuclear lamin, J. Biol. Chem. 277 (2002) 173811738.

[46] M. Izumi, O.A. Vaughan, C.J. Hutchison, D.M. Gilbert, Head and/or CaaX domain deletions of lamin proteins disrupt preformed lamin A and $\mathrm{C}$ but not lamin B structure in mammalian cells, Mol. Biol. Cell 11 (2000) 4323-4337.

[47] E.A. Nigg, G.T. Kitten, K. Vorburger, Targeting lamin proteins to the nuclear envelope: the role of CaaX box modifications, Biochem. Soc. Trans. 20 (1992) 500-504.

[48] K.L. Wilson, The nuclear envelope, muscular dystrophy and gene expression, Trends Cell Biol. 10 (2000) 125-129. 